\title{
Aligning Information System Design and Business Strategy - A Starting Internet Company
}

\author{
Vincent Pijpers, Jaap Gordijn, and Hans Akkermans \\ VU University Amsterdam, FEW/Business Informatics, De Boelelaan 1083a, \\ 1081 HV Amsterdam, The Netherlands \\ $\{$ v.pijpers,gordijn, elly\}@few.vu.nl
}

\begin{abstract}
In this paper we aim to align an organization's information system with its strategic environment. We propose an alignment framework which takes four perspectives into account: Strategy, Value, Processes, IT/IS. This alignment framework is 1) intended for the exploration phase of information system design, 2) considers the complex environment in which an organization - and its IS - operates, and 3) uses conceptual modeling techniques (IS architectures and $e^{3}$ forces) and provides clear steps to analyzes and align the perspectives. We have tested our approach in a real life case setting, where we assisted in aligning an enterprise's IS and business strategy.
\end{abstract}

\section{Introduction}

Although "business strategy" and "Information Systems" (IS) seem to be quite distant topics, their relationship has been of interest for both the academic and business world. Among the first to develop and test frameworks to understand the relationship between business strategy and IT/IS were Henderson and Venkantraman [6] and Luftman et al. 8].

However, these frameworks are limited to only offering fairly abstract, informal facilities to reason about alignment and usually after the information system is already in production. No clear guidelines are provided to practitioners - especially IS practitioners - on how to align both concepts during the design of information systems [3. A second limitation of these traditional frameworks is that they focus on alignment within an organization. How the organization, or more specifically the organization's information system, interacts with its complex environment is traditionally not considered. Yet, nowadays organizations increasingly operate in value webs, which are (complex) collaborations between enterprises to jointly satisfy a consumer need [12, making the interaction with the environment an import concern for business-IT alignment.

To deal with these issues, we propose an alignment framework (see section 2) which 1) can be used during the exploration phase of information system design, 2) provides steps and guidelines for the actual process of alignment and 3) considers the organization's interaction with its complex environment from four different perspectives - "Strategy", "Value", "Process" and "IT/IS" - , each considering a specific concern. Taking multiple perspectives into consideration 
comes however with a price, as it implies that the perspectives should be properly aligned, and not only intra-organizational (eg. strategy and IS within a single organization), but also inter-organizational (eg. interoperability of crossorganizational IS) 4.

Although the proposed alignment framework consider multiple perspectives relevant for business-IT alignment, not all are always interesting to stakeholders, as was the situation with our case study. Therefore we focus in this paper on one specific alignment issue, namely the alignment of an organization's business strategy and IS design. Both business strategy and IS are however rather broad concepts. For instance a business strategy can be expressed in terms of competences, positioning, finances, etc. 7], whereas IS can be expressed in terms of process models, data models, etc. [15. To bring both concepts closer to each other, we operationalize both in terms of "interaction with the environment", which is a well known operationalization in both strategic literature (eg. [1] ) and IS literature (eg. [15]). From a strategic perspective interaction with the environment considers external business forces influencing the actor under investigation on a strategic level (eg. competitors forcing down prices) [11. From a $I S$ perspective, interaction is concerned with the exchange of information with actors in the environment 15 .

To understand and create alignment between a business strategy and IS we use a conceptual modeling approach. For modeling the IS perspective we use basic IS architectures, since they can show how an information system interacts with other actors in its environment (section 3.1). For modeling the strategic perspective we use the $e^{3}$ forces modeling technique [10], since this technique enables us to analyze the strategic environment of an organization in terms of external business forces influencing an organization on a strategic level (section 3.2).

To demonstrate our alignment framework and corresponding alignment approach we present a case study for a start-up Internet enterprise. For over a period of six months we assisted the enterprise with various alignment issues. In the case presented we aim to align the organization's business strategy with the organization's information system, which enables the offering of a valuable service (section 5).

This paper is constructed as follows: First the alignment framework is discussed. Next the modeling techniques used for alignment are presented. Hereafter steps for achieving alignment are discussed. Next the alignment approach is applied on the case study. We end with related work, conclusions and suggestions for further research.

\section{Alignment Framework}

Figure 17a presents the proposed alignment framework. Figure 1] presents the steps to create alignment (see section 4).

Exploration. Before actually implementing information systems it must be understood - on a high level - what the system will do (eg. interact with its environment) and how this will impact the organization. This is vital since mistakes 


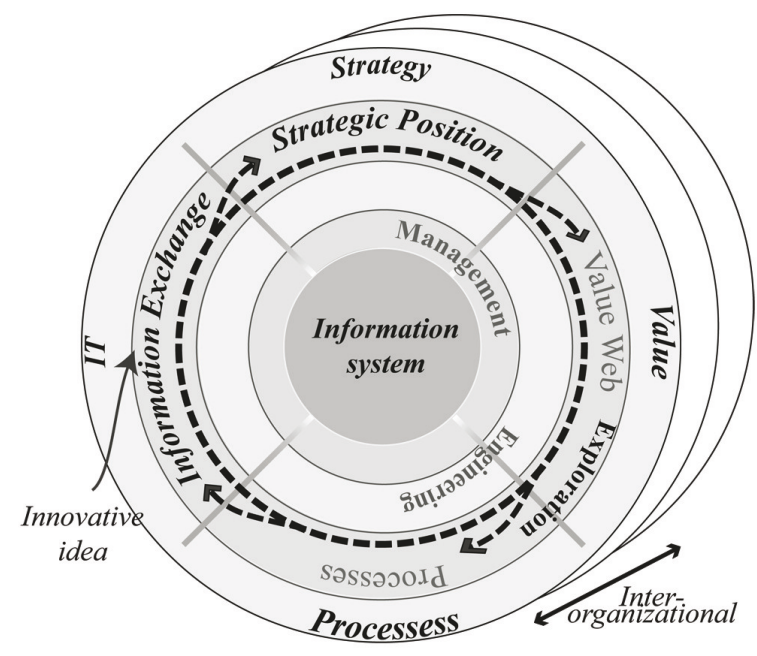

(a) Alignment framework

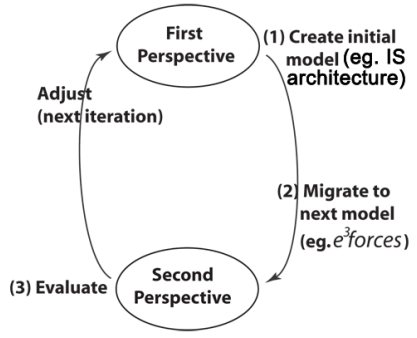

(b) Alignment steps

Fig. 1. Alignment

made in the early phases of information system design can have large (financial) consequences later on [5, 16]. So, the first step is to explore how the information system will interact with its environment from various perspectives (see outer circle alignment model (Fig. 10). Only after the exploration phase is completed, further design (and alignment) should be considered; these additional steps are however outside the scope of this paper.

Multiple perspectives. To explore the interaction of a system/organization with its environment four different perspectives are taken into consideration (see also Fig. 1). Taking various perspectives on the system at hand to separate concerns is well known in traditional requirements engineering (eg. 9]). Although each perspective takes a different viewpoint, they all view the same phenomenon, which is in our case the interaction of a system with its environment. We consider the following perspectives to be relevant: 1) the Business Strategy perspective, which considers how other organizations influence the strategic position of an organization; 2) the Value Creation perspective, which considers how value is created by the value web in which the organization operates; 3) the Processes perspective, which considers the cross-organizational coordination processes to support the value creation; 4) the $I S$ perspective, which considers information systems that interact with their surrounding to exchange information.

We must note that it depends on stakeholders which perspectives are actually explored. Stakeholders can find perspectives irrelevant, simply because they are at that point not (yet) interested in the specific concern explored by a perspective.

Alignment. A consequence of taking multiple perspectives on a system and its interactions with the world is that each perspective should be properly alignment 
with the others. However, alignment between the perspectives should not only be intra-organizational, which considers alignment decisions between perspectives within a single organization [4; alignment should also be created between organizations. This results in two more alignment issues: 1) inter-organizational alignment within a perspective, which considers alignment decisions per single perspective but between multiple organizations (eg. interoperability between cross-organizational IS) and, 2) inter-organizational alignment between perspectives, which considers alignment decisions between perspectives and between multiple organizations (eg. alignment between cross-organizational processes and cross-organizational IS).

Iterative tuning. A naive way to reason about alignment, is to use a kind of top-down or "waterfall" approach as known from traditional software engineering methods. Each perspective would then be developed sequentially, and in a top-down way. We argue that this is, at least for the exploration phase, not a realistic approach. In case studies conducted, innovative ideas came from either the IS perspective (eg. new technologies), process perspective (eg. process optimalization) or value perspective (eg. business opportunities). Also, the world (including the competition) is continuous and fast moving in terms of enterprises, services, and technologies. So, we consider the alignment process a continuous and iterative "tuning" between the four perspectives within an organization and between organizations.

Where to start. If an opportunity for an innovative idea occurs, a basic question is which of the four mentioned perspectives to explore first. In case studies we have performed, we learned that an idea explained by stakeholders initially has a bias to one of the four mentioned perspectives (and actually often toward the IS perspective, as many business ideas stem from technological innovations). Often, this bias is grounded in the stakeholders themselves. We use this biased perspective as the starting point for the exploration of the development of the information system at hand, as stakeholders are familiar with this perspective, and therefore can provide the most information about it. For example, the stakeholders in our case have a technical academic background, so we started with exploring the IS perspective first.

Business Strategy - IS alignment. For the stakeholders in our case study two perspectives were of most interest: the business strategy perspective and the IS perspective. These perspectives were considered most relevant, since the stakeholders sought an IS design which "matched" their business strategy. Subsequently the focus of this paper is on aligning the organization's business strategy and IS by aligning the organization's interaction with its environment as seen from the business strategy perspective and the IS perspective. Understanding an organization's interaction with its environment is a well know construct for both understanding an organization's business strategy (see eg. 11]) and an organization's information systems (see eg. [14,15]). To this end we operationalize both the "business strategy" and "IS" in terms of interaction with the environment, resulting in interactions on a: 
- strategic level, considering the strategic position of the organizations in its "strategic environment", where other organizations influence the organization at hand, thereby determining the success of the organization [11.

- IS level, considering the exchange of information/data between the organization's information system(s) and actors in its "information environment" 15 .

Subsequently, in this paper we aim to design an information system operating in a complex environment, which from a IS perspective enables the offering of a valuable service and from a strategic perspective supports the execution of the organization's business strategy.

\section{$3 \quad$ Alignment Modeling}

Where traditional alignment frameworks offer no concrete methods to address alignment during the design of information systems [3], we take a conceptual modeling-based approach to create alignment within and between organizations. One of the major benefits of utilizing modeling techniques is creating shared understanding over various aspects among stakeholders of the system to be developed 2. In addition, using modeling techniques provides a method to proper elicit the various perspectives on the system to be developed in a structured manner and allows for traceability of changes from one perspective into another 9 . Finally, the models we develop in this exploration phase provide a suitable starting point for the follow-up steps required for designing and implementing the information systems.

Since the focus on this paper is on aligning the strategic environment with the information environment for a system to be developed we take two modeling techniques into account: IS architectures (eg. [1]) and $e^{3}$ forces [10].

\subsection{Information Environment Modeling}

There is a substantial amount of literature on modeling an information system's interaction with its environment (see eg. [15]). Techniques commonly used are UML Use Case Diagrams [14, Data Flow Diagrams and IS architectures (eg. [1]). Since all techniques provide an apt description of an information system's interaction we aim at a notation which is easy and tractable. To this end we have chosen IS architectures. With these models we are interested in identifying two specific aspects: 1) what key technologies are needed for the system at hand, and 2) how the (sub) information systems interact with their environment. Furthermore, based on our field experience, if one of these aspects of the information system changes, chances are high that the business environment will also change.

So for the actor under investigation we model the (sub) information systems and data stores required with squares and rounded squares. Subsequently, we model, via simple arrows, which information is exchanged between the system(s) and actors in its environment (an example can be found in figure 2a). For these 
actors we also model which (sub)-information systems and data stores they require to interact with the actor under investigation. Technologies needed to enable the exchanges are also included (textual), since the selected components reflect important technology choices. For instance in our case study the choice for a GPS or GSM based positioning system had to be made. As we will see, such choices influence the strategic position of an organization.

\subsection{Strategic Environment Modeling}

We use $e^{3}$ forces (see [10]) to model the strategic environment of an organization. As there is to the best of our knowledge no other model-based approach which analyzes the strategic environment of an organization in relation to information system design, the utilization of $e^{3}$ forces to do so is an important contribution of this paper. The $e^{3}$ forces technique provides modeling constructs for representing and analyzing strategic related concepts, such as "strategic position" and "business forces". It enables practitioners to analyze the strategic environment of an organization by analyzing the influence of environmental business forces on a product/service offered by the actor under investigation. The business forces analyzed are directly based on Porter's Five Forces framework [11. In an $e^{3}$ forces model, business forces and their strength are explicitly stated and are related to actors (see figure $2 \mathrm{~b}$ for example). Furthermore, $e^{3}$ forces enables practitioners to quantify a business force's strength such that it is possible to evaluate and compare various alternative strategic positions. Finally, $e^{3}$ forces provides a clear and compact graphical overview of an organization's strategic position and related environmental business forces. The $e^{3}$ forces technique uses the following constructs:

Actor. Actors, modeled as squares, are independent economic (and often also legal) entities [7, which interact with their environment by exchanging objects of value with external business forces [11. Furthermore, an actor has a predetermined business strategy. The business strategy of an organization is the direction and scope of the organization's configuration and position in its environment such that it creates competitive advantage 7 . For an organization to successfully execute its business strategy a matching strategic environment is required [1].

Business Force. Business forces are those organizations that operate in the environment of the actor under study. From a modeling perspective, a business force is not an independent organization but a set of organizations, called market. These external organizations are grouped in markets because by considering sets of organizations we abstract away from the individual and limited influence of many single organizations 11 . This abstraction simplifies the $e^{3}$ forces models to be made, and suffices for the business forces analysis we conduct. Therefore, we consider relationships between actors and specific markets in the actor's environment, rather than the many relationships between actors and each individual organization in the actor's environment. A business force, or market, has a certain strength. The strength of a force indicates to what extent that specific force 
can influence the price and/or configuration of a value object offered to or acquired from an actor. A business force or market is modeled as a layered square. The strength of a business force is expressed by a "strength" arrow. A strength arrow is graphically bundled with the exchange of a value object and points from the business force toward the actor.

Types of Business Forces: Buyer Markets are sets of organizations which are part of the environment of an actor and acquire value objects from the actor under study. Buyer markets influence value objects because they negotiate down prices, bargain for higher quality and, desire different specifications [11. All this is at the expense of the profitability of actor under study [11. Note that we, as described above, do not look at buyers independently, instead we analyze the buyer market of which the individual buyer is part. Supplier Markets, the second business force, are those organizations which provide value objects to actors in the constellation. Suppliers influence value objects provided to actors in a constellation by threatening to alter the configuration of value objects, to increase the price or to limit availability of value objects [11. All this is at the expense of the profitability of the actor under study [11. Competitors, the final business force, are actors that operate in the same industry as the constellation and try to satisfy the same needs of buyers by offering the same value objects to buyer markets as the constellation does [7]. Competitors are a threat for actors because they try to increase their own market share, influence prices and profits and influence customer needs; in short: they create competitive rivalry [11. Due to space limitations we consider "substitutes" and "New Entries" as competitors, which is motivated by the fact that they also try to satisfy the same customer needs.

Determining business force's strength. To analyze the influence of a business force on a value object, $\mathrm{n}$ different aspects $\left(Q_{n}\right)$ need to be analyzed depending on the business force. These aspects are directly derived from the Five Force Model (see [10,11]). To be able to measure and compare the strength of the business force, each of the business aspects related to the business force is scored on a five points scale. The scoring of business aspects is performed with the aid of domain experts. The score " 5 " indicates that the extent to which the business force can influence the value object exchanged is high and " 1 " indicates that it is low. Because the relevance of the aspects can vary per value object exchanged, domain experts give each aspect a weight factor $\left(\beta_{j}\right)$, as done in CBAM [1]. The domain expert have to divide 100 points over the $\mathrm{n}$ aspects $\left(\sum_{j}^{n} \beta_{j}=100\right)$; more points indicate higher relevance. When the weighted expert scores are summed the "strength" of a business force is expressed and indicates to what extent the business force is able to influence the value objects exchanged with the actor.

$$
\text { Strength }_{\text {businessforce }}=\left(\sum_{j}^{n} \beta_{j} * Q_{n}\right) / 5
$$

The total sum is divided by 5 to range buyer market's strength from a maximum "100" to a minimum of "20". For visual purposes a score in the range 
of " $20-48$ " indicates low strength (light gray arrows), "48-76" indicates medium strength (medium gray arrows) and, "76-100" indicates high strength (dark gray arrows).

Value object. Markets and actors in a constellation exchange products and services which are, in generic terms, value objects (see also [5]). A value object has two attributes [11,7]: 1) the configuration consisting of the qualities the object offers and, 2) a price which is expressed in terms of another value object, wanted in return by the provider of the original value object (the price to be paid is usually money, although not obligatory).

\section{Alignment Steps}

To create alignment a number of steps have to be performed (see Fig. 1 b):

Step 0: Preconditions. To align the business strategy of an organization and its IS, we need to know two things: what the business strategy is and what (high level) service the IS will offer (eg. why is the IS needed?). The business strategy will specify how the service offered by the information system is used to create competitive advantage [7]. We consider three generic strategies [7,11]: 1) cost-leadership, which is trying to offer value objects with similar quality as competitors but against a lower price; 2) differentiation, which is to offer value objects with qualities that are unique or differ from competitors; 3) focus, which is focusing on a specific (small) buyer market. What service the IS will offer, is needed to know to elicit what functionalities and technologies the IS will need.

Step 1: Explore First Perspective. As stated, we use the stakeholders' bias to one of the perspectives as a guideline to determine which perspective to explore first. We reason that the stakeholders are most familiar with this perspective, and therefore can provide the most information about it. For example, in the case study at hand the stakeholders were biased toward the IS perspective. Therefore we started with exploring the IS perspective. Subsequently the first step was to create an (initial) IS architecture. With this model we aim to elicit key-technologies used and with what actors (eg. organizations, persons, or even hardware) the system exchanges information, both inbound and outbound.

Step 2: Explore Second Perspective. In step 2 we construct an $e^{3}$ forces model based on the IS architecture from the previous step. The $e^{3}$ forces model will show the strategic interactions whereas the IS architecture shows the information interactions of the system to be designed. For creating the $e^{3}$ forces model we use the following guidelines:

- Actors in the IS architecture are translated into business forces in the $e^{3}$ forces model. Actors providing information become supplier markets. Actors acquiring information become buyer markets. 
- In addition, information exchanges in the IS architecture are translated into value exchanges. We analyze what of value an information exchange represents and what of value is exchanged in return (see also [5]). The basic question answered is: What of value does the information represent for the receiving actor?

- Finally, we model competitors. Information on competitors is not found in the IS architecture, but is required to fully understand the strategic environment of an organization [11. So to complete the $e^{3}$ forces model, we add business forces which offer products/services similar as to offered by the organization under investigation.

Step 3: Evaluate. The next step is to evaluate the strategic interactions by evaluating the various business forces modeled by following the $e^{3}$ forces 's guidelines on how to do so (see sec. 3.2). This evaluation provides us with information on which actors have a large influence on the organization at hand and how (eg. on the service's price or configuration). Subsequently, we can determine if the organization's strategic environment supports the execution of the chosen business strategy. For example, if various actors have a large influence on the price of a service, then a low-cost business strategy might not be the best choice. Note that the analysis performed here heavily relies on business strategy literature by Porter (eg. 11).

Next Iteration. If it appears that the strategic environment does not properly support the execution of the organization's business strategy, then a better strategic environment needs to be found. This is however done by adjusting the information interactions and thus repeating step 1 . We aim to re-position the information system (eg. different technologies) in its environment on an information level such that it still can offer a valuable service to its environment, but from a strategic perspective better supports the execution of the organization's business strategy (which will be evaluated in step 3). To modify the IS architecture in step 1, we present the following guidelines:

- Alternative Resources. If there is a strong supplier force in the strategic environment we try to find alternative sources for the value objects offered by these markets. Translated to the information perspective this means that we try to find different information sources or technologies needed by the information system.

- Alternative Buyers. If there is a strong buyer force we search for other buyer markets to which the service/product can be offered. In the information perspective this means that we try to find new actors which could use the information offered by the information system.

Final design. We basically repeat step 1-3 until we find an IS architecture for the information system which enables the execution of a valuable service and on a strategic level supports the execution of the business strategy chosen by the organization at hand. 


\section{Case Study: Mobzilli - Location Based Advertisement}

Mobzilli, a starting Dutch "Internet" company (www.mobzilli.com), offers the $e$-service "location based advertisement", which offers organizations the possibility to bound advertisements to geographical locations. Potential customers can request the advertisements utilizing a small java application on their mobile phone. So if a customer would be in a shopping street s/he would be able to request the advertisements of the shops in her/his vicinity using her/his mobile phone. Obviously an information system is needed to provision such a service.

We have had intensive contact with Mobzilli for over a period of six months. During these contacts we were not only able to gather information about Mobzilli and its surroundings but we were able to assist in the design of Mobzilli's information system. We consulted Mobzilli during various meetings on aspects ranging from strategic issues to technical issues. In return, they provided us with feedback on our approach and the modeling technique $e^{3}$ forces (eg. what was clear and practicable and what was not).

Step 0: Preconditions. As stated, we need to know the business strategy chosen by Mobzilli to be able to analyze if their strategic environment is desirable. Selecting this strategy itself is not part of out approach, since a business strategy spans multiple years. So, we just ask for the selected strategy. Mobzilli has chosen the business strategy "differentiation", which states that competitive advantage is created by offering a product with a unique configuration but not at low-cost. For the strategic environment of Mobzilli this means that they prefer business forces which do not influence the configuration of their service but which are allowed to influence the price of the service [11.

\subsection{First Iteration}

Step 1: Exploring the Information Perspective. Since Mobzilli's founders had a bias for the IT perspective due to the technical innovation of their idea, we start with exploring the IS perspective. So as a first step, we create an IS architecture. We aim to elicit here not only what information is exchanged between the information system and its environment, but also to elicit a number of technologies used to facilitate these communications. Fig. 2 a shows the IS architecture for Mobzilli's information system initial design.

The IS architecture shows Mobzilli as the central actor incorporating three sub-information systems: 1) a database system, which stores all the ads received from various organizations, 2) an ads generator, which retrieves the ads from the database depending on the location given and forwards the advertisement to the clients mobile, and 3) an analysis system, which serves to provide statistical information of advertisements requested. Furthermore the model shows that 1) a GPS module provides the coordinates of a customer, 2) a GSM with mobile Internet and Java, which is owned by the customer, receives ads, and 3) "Shops" provide ads for the database and retrieve statistical information. We assume a mobile Internet connection to exist and therefore do not include providers. 


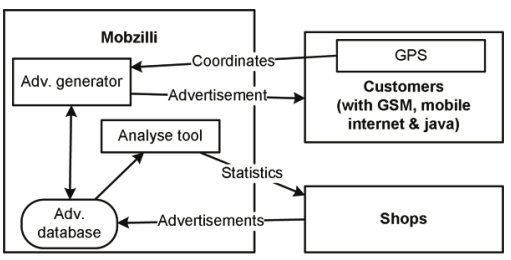

(a) IS architecture

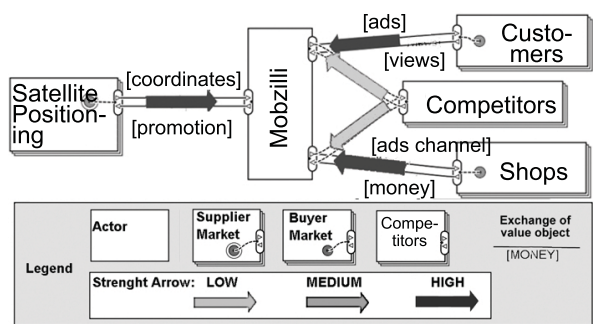

(b) $e^{3}$ forces model

Fig. 2. First Iteration

Step 2: Exploring The Strategic Perspective. In consult with Mobzilli we developed an $e^{3}$ forces model for their initial design to provide insight into their strategic environment (see Fig. 2b).

The $e^{3}$ forces model itself is based on the IS architecture. The actors (organization or sub-system) in the IS architecture are translated into a market that influences Mobzilli. So "Consumers" become the buyer market "Customers", since they acquire a value object from Mobzilli. The actor "Shops" becomes the buyer market "Shops", since they acquire a value object from Mobzilli. Finally, the "GPS" sub-system becomes the supplier market "Satellite Positioning" since the GPS technology supplies valuable information to Mobzilli. In addition, alternative satellite positioning techniques exist (eg. the European Galileo) and the strength of this business force depends on alternative organizations offering the same service.

Based on the information exchanged between Mobzilli and the various business forces, we determined what of value is actually exchanged. For example, the provision of "ads" and acquisition of "Statistical information" by "Shops" is part of the value object "location based advertisement" offered by Mobzilli, which is their main service offered.

Step 3: Evaluate. Together with Mobzilli we applied the metrics described in sections 3.2 , and after a few iterations we found the final scores. According to the supplier metric the score for the supplier market "Satellite Positioning" is 90 , due to an imbalance in the market. The score indicates a strong influence on the value object "position coordinates" offered to Mobzilli. The value object is however free, therefore the strong influences is on the configuration (eg. accuracy) of the value object and not on the price. Furthermore, for Mobzilli to utilize GPS technology each user must have a GPS module. This however limits the amount of possible customers, resulting in a high score of 79 for "Customers". The strength of "Shops" for "Advertisement Channel" is also high (87) since the service is not important for "Shops" (eg. plenty alternatives for advertisement).

Based on these first results it can be concluded that their information system design results in a strategic environment which is not optimal for the execution of their chosen business strategy ("differentiation"). Not only is their a strong supplier force which influences the configuration, there are also two strong buyer 
forces which influence the configuration of Mobzilli's service. Since the service is new the influence of competitors is still low and therefore neglected.

This analysis provided rationale for Mobzilli's feeling that their initial information system design had a strategic environment which does not support the execution of their business strategy, even though the design allows for the service to be provisioned.

\subsection{Second Iteration}

Step 1: Exploring the Information Perspective. Mobzilli focused on specific technology used as a first step to create more alignment between their business strategy and IS. As the first $e^{3}$ forces model shows, using GPS limits the client group size and results in a strong supplier. To this end, alternative methods for client positioning were considered. Mobzilli chose to replace satellite positioning with positioning via triangulation of a GSM's signal strength.

In relation to the original IS architecture, the modified architecture (Fig. 3 a) shows that "GPS" is replaced by a GSM with Java app, which computes the customer's location based on triangulation of signal strengths.

Step 2: Exploring the Strategic Perspective. Based on the second IS architecture an $e^{3}$ forces model was made (see Fig. $3 \mathrm{~b}$ ). The main modification is that the market "Satellite Positioning" is replaced by the market "Position Software". Since the technology to triangulate signal strength from GSM's has to be acquired, "java app" is translated into the market "Position Software".

Step 3: Evaluate. Together with Mobzilli we again determined the strength of the various business forces, and after a few iterations we found the final scores. The score for the supplier market "Position Software" is 80, although still high, it is lower than the score for "Satellite positioning". Again the strong influence is on the configuration (eg. interfaces) and not on the price of the value objects (eg. free licenses exist). Using the metric for buyer markets resulted in a score of 72 for "Customers" and 87 for "Shops". Note that the score for "Shops" has remained the same, but the score of "Customers" decreased. This is the result of more customers without GPS. Therefore, there is only one strong force on the buyer side remaining ("Shops") which can make demands in regard to the

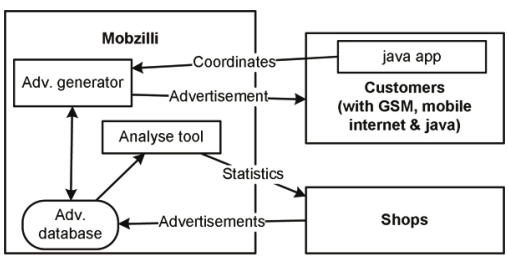

(a) IS architecture

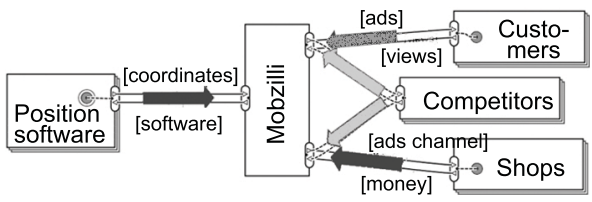

(b) $e^{3}$ forces model

Fig. 3. Second Iteration 
configuration of Mobzilli's service. Since the service is still new the influence of competitors is still low and therefore not relevant.

The strategic environment is still not optimal for the execution of the chosen business strategy. The scores of the various forces are however better than in the initial design. This indicates that the revised information environment of Mobzilli's information system is better then in the first iteration. This notion was supported by Mobzilli. However, Mobzilli wondered if an even better strategic environment could be found, so again revisions were made.

\subsection{Final Iteration}

Step 1: Exploring the Information Perspective. Mobzilli chose to keep using GSM triangulation technology. However, Mobzilli did consider a new group of users for which their "location based advertisement" service is valuable: "Musea". The final IS architecture (Fig. 4a) again does not show many difference in regard to the previous IS architecture, yet an additional actor is modeled "Musea", which has the same interaction with the system as "Shops".

Step 2: Exploring the Strategic Perspective. The $e^{3}$ forces model for the strategic environment is presented in figure 4b. The model is based on the final IS architecture and shows a new buyer market ("Musea"), which acquires the same value object from Mobzilli as "Shops" does.

Step 3: Evaluate. In cooperation with Mobzilli the various metrics were applied. The score for "Positioning Software" (80) remained the same. Using the metric for buyer markets resulted in a score of 69 for 'Customers", the score decreased since again an even larger population exists. The new score for "Shops" is 78 . By adding a market, more trading areas for Mobzilli are available and thereby the strength of "Shops" decreased. For "Musea" the scores is 65. The strength of "Musea" is less than that of "Shops" because less alternatives are at hand for this buyer market. With this new design only one strong force remains. Since the service is still new, also for the new buyer market, the influence of competitors is still low and therefore not relevant.

Analysis Conclusion. It was concluded that the final design provides a strategic environment which is acceptably aligned with the system's final information

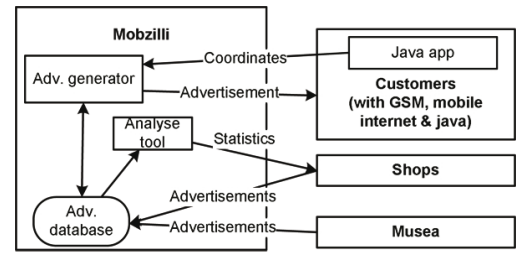

(a) IS architecture

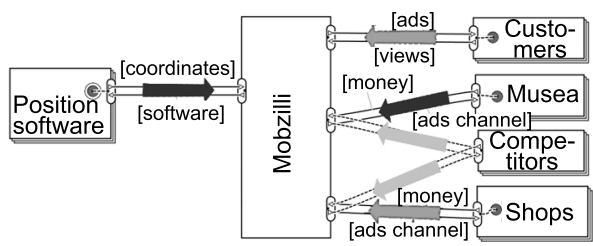

(b) $e^{3}$ forces model

Fig. 4. Final Iteration 
environment. This notion was supported by Mobzilli. By carefully adjusting the IS architecture, based on findings from the strategic environment, an environment was found which has as minimal as possible influence on the configuration of Mobzilli's service and subsequently allows for the execution of the chosen business strategy. Although Mobzilli often had a feeling concerning various design choices (eg. which technology). They were however unable to properly related technical issues to their business strategy. With the aid of our alignment approach we were able to provide Mobzilli with theoretical rationale for their design choices.

\section{Related Work}

The most relevant related work it that of Thevenet and Salinesi [13. Their method, INtenional STrategic Alignment (INSTAL), analyzes organizations at two levels: the strategic level and the operational level. Using documentation from both levels a third level is created where the synergy between both levels is documented, both the strategic and operational level are modeled within one single model [13. However, in contrast to our approach, which has an external view of organizations, INSTAL has an internal view on organizations. Furthermore, modeling both strategic and operations aspects within one model might cause confusion since both viewpoints highlight quite different aspects of organizations.

\section{Conclusion}

The aim of this paper was to align an organization's business strategy and information system design within a complex environment. We accomplished this by analyzing - and subsequently aligning - the organization's interactions from a strategic perspective and information perspective. Furthermore, we provided clear steps on how to explore and align both perspectives. The application of our methodology in a real-life setting showed that we are able to align an organization's IS interactions on an informational level and the organization strategic interactions. This alignment resulted in a IS design which enables the provision of a valuable service and interacts with its environment such that the corresponding strategic interactions allow for the execution of the organization's business strategy. However, as our alignment framework indicates, various other alignment issues have to be considered during the exploration phase of information system design, leaving room for further research.

Acknowledgments. The authors wish to thank E. Dubois, J. A. Pastor Collado, J. Falcão e Cunha, M. Léonard, C. Salinesi, A. Persson, L. Patrcio, N. Castell, and P. Botella for discussions about the framework in Fig. 1 and W. Chowanski and R. Ladchartabi from Mobzilli for providing case material. This work has been partly sponsored by NWO project COOP 600.065.120.24N16. 


\section{References}

1. Asundi, J., Kazman, R., Klein, M.: Using economic considerations to choose amongst architecture design alternatives. Technical report, Software Engineering Institute (2001)

2. Borst, W.N., Akkermans, J.M., Top, J.L.: Engineering ontologies. International Journal of Human-Computer Studies 46, 365-406 (1997)

3. Chan, Y., Horner Reich, B.: IT alignment: what have we learned? Journal of Information Technology 22, 297-315 (2007)

4. Derzsi, Z., Gordijn, J.: A framework for business/IT alignment in networked value constellations. In: Latour, T., Petit, M. (eds.) Proceedings of the workshops of the 18th International Conference on Advanced Information Systems Engineering (CAiSE 2006), Namur, B, pp. 219-226. Namur University Press (2006)

5. Gordijn, J., Akkermans, H.: Value based requirements engineering: Exploring innovative e-commerce idea. Requirements Engineering Journal 8(2), 114-134 (2003)

6. Henderson, J., Venkantraman, N.: Strategic alignment, leveraging information technology for transforming organizations. IBM systems journal (1) (1993)

7. Johnson, G., Scholes, K.: Exploring Corporate Strategy. Pearson Education Limited, Edinburgh (2002)

8. Luftman, J., Papp, R., Brier, T.: The strategic alignment model: Assessment and validation. In: Proceedings of the Information Technology Management Group of the Association of Management (AoM) 13th annual International Conference, Vancouver, pp. 57-66 (1995)

9. Nuseibeh, B., Kramer, J., Finkelstein, A.: A framework for expressing relationships between multiple views in requirements specification. IEEE Transactions on Software Engineering 20(10), 760-773 (1994)

10. Pijpers, V., Gordijn, J.: e3forces: Understanding strategies of networked e3value constellation by analyzing environmental forces. In: Proceedings of the 19th Conference on Advanced Information System Engineering 2007. LNCS, vol. 4495, pp. 188-203. Springer, Heidelberg (2007)

11. Porter, M.E.: Competitive advantage. Creating and sustaining superior performance. The Free Press, New York (1980)

12. Tapscott, D., Ticoll, D., Lowy, A.: Digital Capital - Harnessing the Power of Business Webs. Harvard Business School Press, Boston (2000)

13. Thevenet, L.H., Salinesi, C.: Aligning is to organization's strategy: The instal method. In: Krogstie, J., Opdahl, A.L., Sindre, G. (eds.) CAiSE 2007 and WES 2007. LNCS, vol. 4495, pp. 203-217. Springer, Heidelberg (2007)

14. UML. Uml 2.0, www.uml.org

15. Wieringa, R.J.: Design Methods for Reactive Systems. Morgan Kaufman Publishers, San Francisco (2003)

16. Yu, E.: Models for supporting the redesign of organizational work. In: COCS 1995: Proceedings of conference on Organizational computing systems, pp. 226236. ACM Press, New York (1995) 